ARTICLE

DOI: $10.1038 / \mathrm{s} 41467-017-02310-9$

\title{
A pH-gated conformational switch regulates the phosphatase activity of bifunctional HisKA-family histidine kinases
}

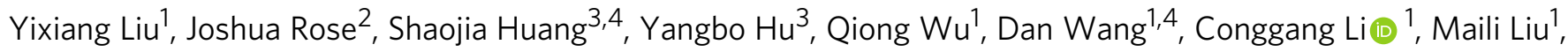
Pei Zhou (1) ${ }^{2}$ \& Ling Jiang ${ }^{1}$

Histidine kinases are key regulators in the bacterial two-component systems that mediate the cellular response to environmental changes. The vast majority of the sensor histidine kinases belong to the bifunctional HisKA family, displaying both kinase and phosphatase activities toward their substrates. The molecular mechanisms regulating the opposing activities of these enzymes are not well understood. Through a combined NMR and crystallographic study on the histidine kinase HK853 and its response regulator RR468 from Thermotoga maritima, here we report a $\mathrm{pH}$-mediated conformational switch of HK853 that shuts off its phosphatase activity under acidic conditions. Such a $\mathrm{pH}$-sensing mechanism is further demonstrated in the EnvZ-OmpR two-component system from Salmonella enterica in vitro and in vivo, which directly contributes to the bacterial infectivity. Our finding reveals a broadly conserved mechanism that regulates the phosphatase activity of the largest family of bifunctional histidine kinases in response to the change of environmental $\mathrm{pH}$.

\footnotetext{
${ }^{1}$ Key Laboratory of Magnetic Resonance in Biological Systems, State Key Laboratory of Magnetic Resonance and Atomic and Molecular Physics, National Center for Magnetic Resonance in Wuhan, Wuhan Institute of Physics and Mathematics, Chinese Academy of Sciences, Wuhan 430071, China.

${ }^{2}$ Department of Biochemistry, Duke University Medical Center, Durham, NC 27710, USA. ${ }^{3}$ CAS Key Laboratory of Special Pathogens and Biosafety, Center for Emerging Infectious Diseases, Wuhan Institute of Virology, Chinese Academy of Sciences, Wuhan 430071 China. ${ }^{4}$ University of Chinese Academy of Sciences, Beijing 100049 China. Yixiang Liu and Joshua Rose contributed equally to this work. Correspondence and requests for materials should be addressed to L.J. (email: lingjiang@wipm.ac.cn)
} 
S ignal transduction by the two-component system (TCS) proteins is the primary mechanism for bacteria to detect environmental changes and orchestrate cellular responses ${ }^{1-3}$. Such a process also plays a critical role in bacterial pathogenesis, for example, by mediating pathogenicity genes during the host infection of Salmonella in response to the acidification of the phagocytic vacuole ${ }^{4}$.

A typical TCS contains a histidine kinase (HK) and a response regulator (RR). HKs are homodimers consisting of an extracellular sensor domain, a transmembrane connector, and a cytoplasmic portion $\left(\mathrm{HK}^{\mathrm{cp}}\right)$ that contains the catalytic activity (Fig. 1a). The cytoplasmic portion of histidine kinases can be further divided into the dimerization and histidine-containing phosphotransfer (DHp) domain and the catalytic and ATP binding (CA) domain ${ }^{1,5}$. About $80 \%$ of all sensor kinases belong to the HisKA family ${ }^{6}$, including HK853 from Thermotoga maritima, EnvZ from Salmonella, and others.

After sensing the environmental stimuli, the histidine kinase autophosphorylates a conserved histidine residue in the $\mathrm{DHp}$ domain (e.g., H260 of HK853; Fig. 1a) by its catalytic CA domain, which then transfers the phosphoryl group to an aspartate residue of the receiver domain of its cognate response regulator (RR) (e.g., D53 of RR468; Fig. 1a). Such an event triggers the interaction of the phosphorylated RR (phospho-RR) with downstream genes or protein targets for regulation of a variety of cellular functions $s^{1,7}$.
Due to the essential role of the phosphorylated response regulator in orchestrating the cellular response, its phosphorylation level is tightly controlled. Such a regulation is predominantly achieved through the opposing kinase and phosphatase activities embedded within the module of bifunctional histidine kinase. The conserved histidine residue involved in the phosphoryl transfer process of the DHp domain has also been implicated in the dephosphorylation reaction; the phosphatase activity is additionally affected by conserved Thr and Asn residues of the E/ DxxT/N motif (e.g., T264 of HK853; Fig. 1a) immediately adjacent to the conserved histidine residue ${ }^{8,9}$. How a bifunctional histidine kinase switches its kinase and phosphatase activities on and off to maintain a balanced phosphorylation level of the response regulator has remained poorly understood.

Here we report a molecular mechanism that gates the phosphatase activity of the bifunctional histidine kinase HK853, a member of the HisKA family, from Thermotoga maritima. Our study reveals a $\mathrm{pH}$-mediated conformational change, involving a sidechain rotameric switch of the catalytic histidine residue that inactivates the phosphatase activity of HK853. Such a mechanism similarly mediates the phosphatase activity of the classical EnvZ bifunctional histidine kinase, also of the HisKA family, from Salmonella enterica in vitro; accordingly, the transcriptional response genes of the EnvZ/OmpR TCS are upregulated at low $\mathrm{pH}$ in cells, consistent with the $\mathrm{pH}$-gated inactivation of the EnvZ

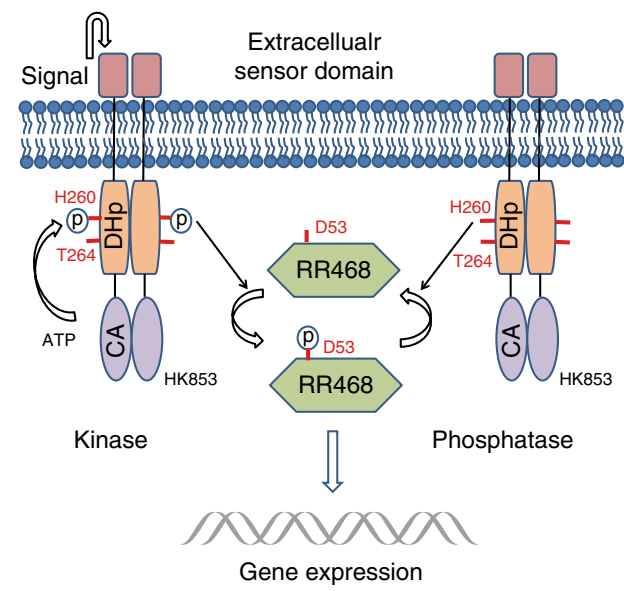

C

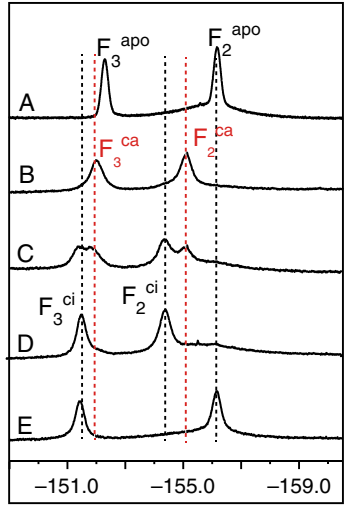

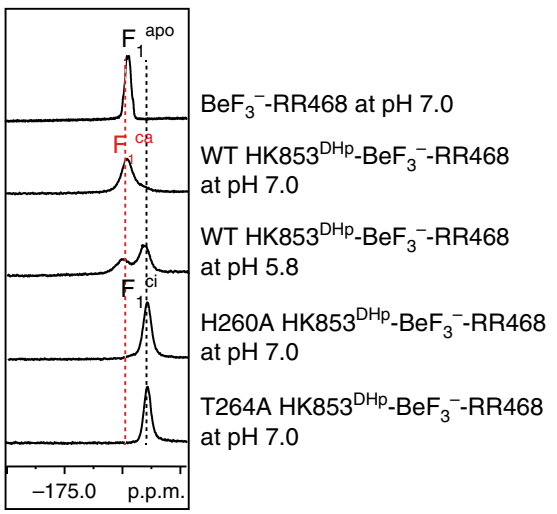

b $\mathrm{BeF}_{3}{ }^{-}{ }^{-\mathrm{RR}} 468$

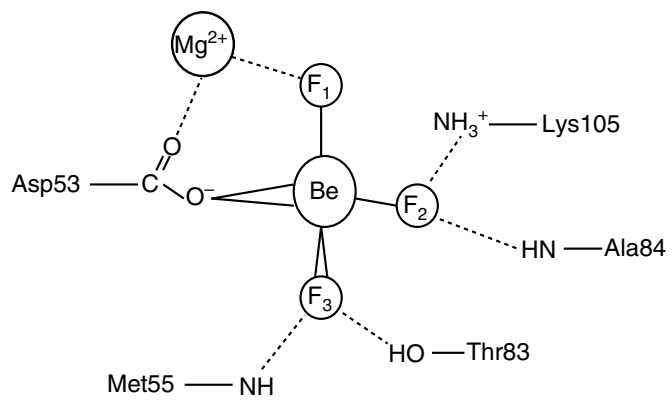

d

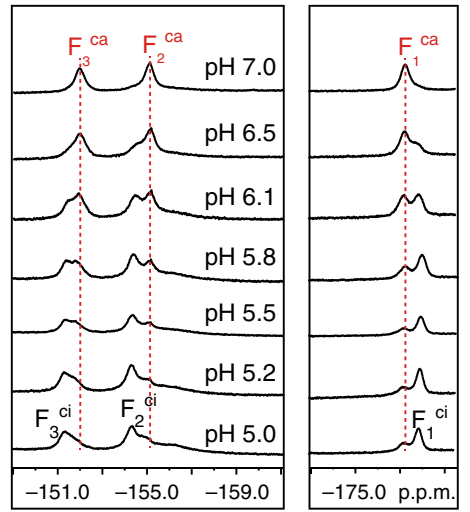

Fig. $1^{19} \mathrm{~F}$ NMR studies reveal multiple conformational states of the $\mathrm{HK} 853-\mathrm{BeF}_{3}{ }^{-}$-RR468 protein complex. a The signal transduction pathway of the twocomponent system, exemplified by the bifunctional histidine kinase, HK853, and the downstream response regulator, RR468. b A schematic illustration of the coordination of $\mathrm{BeF}_{3}{ }^{-}$in the active site of $\mathrm{BeF}_{3}{ }^{-}$-RR468 (PDB 3GL9). $\mathbf{c}$ The ${ }^{19} \mathrm{~F} \mathrm{NMR} \mathrm{spectra} \mathrm{of} \mathrm{BeF}_{3}{ }^{-}-\mathrm{RR} 468$ without and with $\mathrm{HK} 853^{\mathrm{DHp}}$ and mutants: spectrum $\mathrm{A}, \mathrm{BeF}_{3}{ }^{-}-\mathrm{RR} 468$ at $\mathrm{pH}$ 7.0; spectrum $\mathrm{B}$, the wild-type $\mathrm{HK} 853^{\mathrm{DHp}} \mathrm{BeF}_{3}{ }^{-}$-RR468 complex at pH 7.0; spectrum C, the wild-type complex at pH 5.8; spectrum $\mathrm{D}$, the $\mathrm{H} 260 \mathrm{~A} \mathrm{HK} 853^{\mathrm{DHp}}-\mathrm{BeF}_{3}{ }^{-}$-RR468 complex at $\mathrm{pH} 7.0$; spectrum $\mathrm{E}$, the T264A HK853 ${ }^{\mathrm{DHp}}-\mathrm{BeF}_{3}{ }^{-}{ }^{-} \mathrm{RR} 468 \mathrm{complex}$ at $\mathrm{pH} 7.0$. Fluorine signals

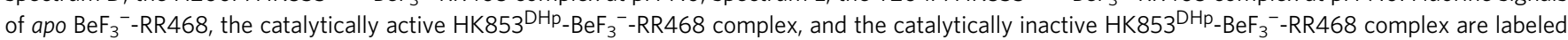
as $\mathrm{F}^{\mathrm{apo}}, \mathrm{F}^{\mathrm{ca}}$, and $\mathrm{F}^{\mathrm{ci}}$, respectively. $\mathbf{d}$ The ${ }^{19} \mathrm{~F}$ NMR spectra of the wild-type $\mathrm{HK} 853^{\mathrm{DHp}}-\mathrm{BeF}_{3}{ }^{-}$- $\mathrm{RR} 468$ complex at different $\mathrm{pH}$ conditions 
phosphatase activity and the subsequent accumulation of the phospho-OmpR. We suggest that the regulatory mechanism revealed by this study may represent a universal $\mathrm{pH}$ sensor utilized by the largest family of bifunctional histidine kinases in bacteria and other organisms to detect and respond to the change of the host environment to establish pathogenic infection.

\section{Results}

${ }^{19} \mathrm{~F}$ NMR reveals two conformational states of the complex. $\mathrm{BeF}_{3}{ }^{-}$, a well-established phosphoryl analog for studying phosphorylated proteins, has been previously used to generate the phosphorylation mimic of the response regulator ${ }^{10-12}$. Using a similar approach, we generated the $\mathrm{BeF}_{3}{ }^{-}$-conjugated form of RR468, the cognate response regulator of HK853 from Thermotoga maritima, with the beryllium atom forming a covalent bond with the phosphate-accepting residue D53 and with three fluorine atoms (marked as $F_{1}, F_{2}$, and $F_{3}$ in Fig. $1 b$ ) interacting with the $\mathrm{Mg}^{2+}$ ion, sidechains of K105 and T83, and amide groups of M55 and A84 of RR468 ${ }^{5}$. When RR468 was mixed with an excess amount of $\mathrm{BeF}_{3}^{-}$, we were able to detect three broad fluorine signals from the protein-bound $\mathrm{BeF}_{3}{ }^{-}$in the active site of RR468 between -180.0 p.p.m. and -150.0 p.p.m. (Fig. 1c, spectrum A), which were distinct from the strong and narrow signals of the unbound beryllium fluoride molecules ${ }^{13,14}$.

The identities of the protein-bound fluorine signals were assigned based on the solvent-induced isotopic shift (SIIS) experiment ${ }^{15-17}$ (Supplementary Fig. 1): the upper field ${ }^{19} \mathrm{~F}$ signal at -177.18 p.p.m. was the least sensitive to $\mathrm{D}_{2} \mathrm{O}$ exchange and was identified as the fluorine atom $\mathrm{F}_{1}$ that coordinates $\mathrm{Mg}^{2+}$; the downfield ${ }^{19} \mathrm{~F}$ signal at -152.13 p.p.m. displayed the largest shift when the water $\left(\mathrm{H}_{2} \mathrm{O}\right)$ solvent was replaced with $100 \% \mathrm{D}_{2} \mathrm{O}$ (Supplementary Table 1 and Supplementary Fig. 1) and, based on the large SIIS effect, was assigned to fluorine $F_{3}$ that forms hydrogen bonds with the side chain of T83 and the amide group of M55; the central signal at -156.01 p.p.m. experienced an intermediate SIIS effect and was assigned to fluorine $F_{2}$ that forms a salt bridge with the sidechain of K105 and a hydrogen bond with the amide group of A84. The identity of this fluorine atom was further validated by the lysine sidechain specific $\mathrm{H} 3(\mathrm{~N}) \mathrm{F}$ experiment that correlated the $\mathrm{F}_{2}$ fluorine signal with the terminal $\mathrm{NH}_{3}$ proton of K105 (Supplementary Fig. 2).

After identifying individual fluorine signals in $\mathrm{BeF}_{3}{ }^{-}$-RR468, we examined the fluorine signals of $\mathrm{BeF}_{3}{ }^{-}$-RR468 in complex with the DHp domain of HK853 (HK853 ${ }^{\mathrm{DHp}}$ ) that possesses both the kinase and phosphatase activities. The DHp domain of HK853 forms a homodimer and interacts with RR468 at an equal molar ratio ${ }^{5}$. During the titration of $\mathrm{HK} 853^{\mathrm{DHp}}$ at neutral $\mathrm{pH}$, all of the signals in the ${ }^{19} \mathrm{~F}$ spectra became broadened, and a set of new peaks emerged downfield of the original ones, indicating the formation of the $\mathrm{HK} 853^{\mathrm{DHp}}-\mathrm{BeF}_{3}{ }^{-}-\mathrm{RR} 468$ complex (Fig. 1c, spectrum B). Among the three fluorine signals, $F_{2}$ was the most perturbed signal with a chemical shift difference of 1.2 p.p.m., indicating that the interaction of the $\mathrm{HK} 853^{\mathrm{DHp}}$ domain significantly alters the salt bridge between $\mathrm{F}_{2}$ and the sidechain of K105.

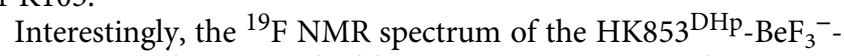
RR468 complex was highly sensitive to $\mathrm{pH}$ changes, a phenomenon that was not observed in $\mathrm{BeF}_{3}{ }^{-}$-RR468 alone. When $\mathrm{pH}$ decreased from 7.0 to 5.0, an additional set of three fluorine signals emerged in the ${ }^{19} \mathrm{~F}$ NMR spectra (Fig. 1c, spectrum $C$ and Fig. 1d). These new signals were located next to the original ones and showed higher intensities when the $\mathrm{pH}$ dropped below 6.0, indicating a slow exchange between two distinct conformations of the $\mathrm{HK} 853^{\mathrm{DHp}}-\mathrm{BeF}_{3}{ }^{-}-\mathrm{RR} 468$ complex. For clarity, we designate the signals of free $\mathrm{BeF}_{3}{ }^{-}$-RR468 as $\mathrm{F}_{x}$ apo $(x=1,2,3)$, the signals of the HK853 ${ }^{\mathrm{DHp}}-\mathrm{BeF}_{3}{ }^{-}$-RR468 complex at $\mathrm{pH} 7.0$ as $\mathrm{F}_{x}^{\mathrm{ca}}(x=1,2,3)$, and the signals under acidic conditions ( $\mathrm{pH} 5.0)$ as $\mathrm{F}_{x}{ }^{\mathrm{ci}}(x=1,2,3)$ (Fig. 1b-d). It is important to note that the circular dichroism spectra of $\mathrm{HK} 853^{\mathrm{cp}}$ and its complex with RR468 remained unchanged between $\mathrm{pH} 5.0$ and 8.0 , suggesting that the observed changes of the fluorine signals were not caused by denaturation of the proteins under acidic conditions (Supplementary Fig. 3).

$\mathrm{H} 260$ is involved in the conformational exchange. $\mathrm{A} \mathrm{pH}$ dependent protein conformational change typically occurs in response to the change of the protonation state of a $\mathrm{pH}$-sensitive residue, such as a histidine residue. As the conserved autophosphorylated histidine residue of the bifunctional histidine kinases acts as both the phosphate donor in the kinase reaction and the catalytic residue in the phosphatase reaction ${ }^{18,19}$, we generated the corresponding $\mathrm{H} 260 \mathrm{~A}$ mutation of the $\mathrm{DHp}$ domain of HK853 (HK853 ${ }^{\mathrm{DHp}}$ ) and verified that this mutation indeed displayed diminished phosphatase activity ${ }^{5}$ (Supplementary Fig. 4; uncropped images shown in Supplementary Fig. 5). Surprisingly, the ${ }^{19} \mathrm{~F}$ NMR spectrum of the H260A mutant of the $\mathrm{HK} 853^{\mathrm{DHP}}-\mathrm{BeF}_{3}{ }^{-}-\mathrm{RR} 468$ complex displayed only three proteinbound fluorine signals that matched the chemical shifts of the $\mathrm{F}_{x} \mathrm{ci}$ signals observed in the wild-type protein complex under acidic conditions (Fig. 1c, compare spectra C and D). Furthermore, these fluorine signals no longer displayed $\mathrm{pH}$-dependent changes (Supplementary Fig. 6). Additionally, the signals of the H260A HK853 ${ }^{\mathrm{DHp}}-\mathrm{BeF}_{3}{ }^{-}$-RR468 complex were much sharper than the signals from the wild-type complex: the half-width of the $F_{1}$ signal in the wild-type complex was $306 \mathrm{~Hz}$ at $\mathrm{pH} \mathrm{7.0,} \mathrm{whereas}$ the half-width of the same signal in the H260A mutant complex was only $220 \mathrm{~Hz}$; such an observation supports the notion that the $\mathrm{pH}$-dependent conformational exchange mediated by $\mathrm{H} 260$ is suppressed or eliminated in this mutant.

An inactive phosphatase conformation of $\mathrm{HK853}$ at low $\mathrm{pH}$. As our ${ }^{19} \mathrm{~F}$ NMR studies revealed the presence of a second conformation that is marginally populated at neutral $\mathrm{pH}$, but becomes the predominant state under acidic conditions, we determined the crystal structure of $\mathrm{HK} 853^{\mathrm{cP}}$ and its cognate response regulator $\mathrm{RR} 468$ in the presence of the phosphoryl analog $\mathrm{BeF}_{3}{ }^{-}$and ATP analog, AMPPNP, under acidic conditions ( $\mathrm{pH}$ 5.0) in order to visualize this minor conformation at neutral $\mathrm{pH}$. The crystal structure, determined at $2.68 \AA$ resolution (Table 1, a portion of the electron density map is shown in Supplementary Fig. 7), reveals a heterotetrameric complex consisting of two $\mathrm{HK} 853^{\mathrm{cP}}$ and two $\mathrm{BeF}_{3}{ }^{-}$-RR468. The two antiparallel helices of the $\mathrm{N}$-terminal $\mathrm{DHp}$ domains pack into a central four-helix bundle, with the C-terminal CA domains and the RR468 monomers docking onto and surrounding the central four-helix bundle (Fig. 2a). The AMPPNP is captured in the hydrolyzed form of ADP, as previously observed ${ }^{5}$. The structure of the $\mathrm{HK} 853^{\mathrm{CP}}-\mathrm{BeF}_{3}{ }^{-}$-RR468 complex is close to symmetric, with the backbone rmsds of 0.4 and $0.2 \AA$ between individual monomers of the kinase and response regulator, respectively. The overall architecture of the $\mathrm{HK} 853^{\mathrm{CP}}-\mathrm{BeF}_{3}{ }^{-}$-RR468 complex crystallized at $\mathrm{pH} 5.0$ is similar to the previously reported structure of the HK853 ${ }^{\mathrm{cp}}$-RR468 complex determined at higher $\mathrm{pH}(\mathrm{pH}$ 5.6; PDB 3DGE) $)^{5}$.

However, a close examination of the conserved and catalytically important histidine residue involved in the $\mathrm{pH}$-dependent change of NMR signals reveals distinct conformations. In the previously reported structure of the $\mathrm{HK} 853^{\mathrm{cP}}-\mathrm{RR} 468$ complex determined at higher $\mathrm{pH}$, the sidechain of $\mathrm{HK} 853^{\mathrm{cP}} \mathrm{H} 260$ adopts a trans $\chi^{1}$ rotameric conformation and points toward D53, the 
Table 1 Data collection and refinement statistics for the wild-type and T264A HK853 ${ }^{\mathrm{CP}_{-}} \mathrm{BeF}_{3}{ }^{-}-\mathrm{RR}$ (68 complex $^{\mathrm{a}}$

\begin{tabular}{|c|c|c|}
\hline & $\begin{array}{l}\text { Wild-type HK853 } \\
\mathrm{BeF}_{3}{ }^{-}-\mathrm{RR} 468\end{array}$ & $\begin{array}{l}\text { T264A HK853 }^{\mathrm{CP}}{ }_{-} \\
\mathrm{BeF}_{3}{ }^{-}-\mathrm{RR} 468\end{array}$ \\
\hline \multicolumn{3}{|l|}{ Data collection } \\
\hline Space group & C 121 & C 121 \\
\hline \multicolumn{3}{|l|}{ Cell dimensions } \\
\hline$a, b, c(\AA)$ & $177.97,98.38,72.05$ & $178.46,98.34,71.46$ \\
\hline$\alpha, \beta, \gamma\left({ }^{\circ}\right)$ & $90.0,110.5,90.0$ & $90.0,109.8,90.0$ \\
\hline Resolution $(\AA)$ & $49.19-2.68(2.78-2.68)$ & $\begin{array}{l}45.50-3.63 \\
(3.76-3.63)\end{array}$ \\
\hline$R$-meas & $0.081(0.886)$ & $0.165(0.614)$ \\
\hline$|/ \sigma|$ & $20.73(3.14)$ & $6.56(2.41)$ \\
\hline Completeness (\%) & $100(100)$ & $100(97)$ \\
\hline Unique reflections & $32,771(3254)$ & $13,230(1272)$ \\
\hline Redundancy & $7.7(7.7)$ & $4.2(4.0)$ \\
\hline \multicolumn{3}{|l|}{ Refinement } \\
\hline Resolution $(\AA)$ & $49.19-2.68(2.78-2.68)$ & $\begin{array}{l}45.50-3.63 \\
(3.76-3.63)\end{array}$ \\
\hline Unique reflections & $32,762(3254)$ & $13,230(1272)$ \\
\hline$R_{\text {work }} / R_{\text {free }}$ & $0.182 / 0.222$ & $0.208 / 0.240$ \\
\hline No. of atoms & 5714 & 5462 \\
\hline Protein & 5499 & 5398 \\
\hline Ligand/ion & 125 & 64 \\
\hline Water & 90 & 0 \\
\hline Average $B$-factors & 64.55 & 103.22 \\
\hline Protein & 64.45 & 103.16 \\
\hline Ligand/ion & 77.24 & 108.27 \\
\hline Water & 53.23 & \\
\hline \multicolumn{3}{|l|}{ R.m.s. deviations } \\
\hline Bond lengths $(\AA)$ & 0.005 & 0.002 \\
\hline Bond angles $\left({ }^{\circ}\right)$ & 0.81 & 0.56 \\
\hline \multicolumn{3}{|l|}{ Ramachandran } \\
\hline Favored (\%) & 95.6 & 93.8 \\
\hline Outliers (\%) & 0.7 & 0.3 \\
\hline
\end{tabular}

receiving residue of the phosphate group of the response regulator RR468; the imidazole ring of $\mathrm{H} 260$ of $\mathrm{HK} 853^{\mathrm{cP}}$ is located within $3.5 \AA$ of a sulfate molecule that mimics the phosphate moiety of the phosphorylated D53; and the overall configuration represents a catalytically active conformation. In contrast, in the structure of the $\mathrm{HK} 853^{\mathrm{CP}}-\mathrm{BeF}_{3}{ }^{-}$- RR468 complex crystallized at lower $\mathrm{pH}(\mathrm{pH}$ 5.0), where the conformational state in solution is predominantly the $\mathrm{F}^{\mathrm{ci}}$ state, the sidechain of $\mathrm{H} 260$ adopts a gauche- $\chi^{1}$ rotameric conformation (Supplementary Fig. 7), with the imidazole ring of $\mathrm{H} 260$ located at $11.2 \AA$ away from the beryllium atom of the $\mathrm{BeF}_{3}{ }^{-}$-modified D53 of the response regulator RR468; such a configuration would prevent H260 from engaging in the phosphatase activity of HK853 and hence represents a catalytically inactive state (Fig. 2b). Accompanying the sidechain movement of $\mathrm{H} 260$, the inter-domain arrangement of the inactive complex also changed. The $\mathrm{N}$ terminal half of the first helix, which contains H260, was less bent compared to that of the catalytically active structure solved at a higher $\mathrm{pH}$, with the location of the $\mathrm{N}$-terminus of the helix shifting about 2.1 $\AA$. Such a difference in the packing of the DHp domain caused a clockwise rotation of the C-terminal CA domain and the response regulator RR468 around the central helices, resulting in distance shifts between the catalytically inactive and active conformations of as large as $\sim 6.9$ and $\sim 2.7 \AA$ for the CA domain and RR468, respectively (Fig. 2c, d). These structural observations provide a molecular explanation for the lack of the phosphatase activity of HK853 under acidic conditions shown in the Phos-tag assay (Fig. 2e, f; uncropped images shown in Supplementary Fig. 8a, b, respectively) and suggest that the phosphatase activity of $\mathrm{HK} 853$ is regulated by a $\mathrm{pH}$-gated conformational switch.

T264 is required for the H260-mediated phosphatase activity. Beside H260, T264 is another conserved residue that is critical for the transmitter phosphatase activity of HK853. When we titrated the T264A mutant of $\mathrm{HK} 853^{\mathrm{DHp}}$ into the $\mathrm{BeF}_{3}{ }^{-}$RR468 solution, the fluorine signal $\left(\mathrm{F}_{2}\right)$ interacting with K105 of RR468 was unchanged; however, the other two fluorine signals interacting with T83 and $\mathrm{Mg}^{2+}$ of RR468 were perturbed and were in good agreement with the fluorine signals of the inactive $\mathrm{F}^{\mathrm{ci}}$ state of the HK853-RR468 complex (Fig. 1c, spectrum E). These observations suggest that the T264A HK853 mutant still formed a complex with the response regulator RR468, but the critical chemical entity participating in the RR468 K105-fluorine $\left(\mathrm{F}_{2}\right)$ interaction was absent in the T264A mutant of the HK853RR468 complex.

As T264 of $\mathrm{HK} 853^{\mathrm{cp}}$ is at least $5 \AA$ away from either K105 or the K105-interacting fluorine atom $\left(\mathrm{F}_{2}\right) \mathrm{Of} \mathrm{BeF}_{3}{ }^{-}$-modified D53, we suggest that the chemical entity causing the chemical shift perturbation of the K105-interacting fluorine atom $\left(\mathrm{F}_{2}\right)$ is a T264-mediated water molecule, which in turn forms a hydrogen bond with the $F_{2}$ fluorine atom; furthermore, this water molecule is likely the catalytic water molecule that additionally interacts with $\mathrm{H} 260$ of HK853 in the trans $\chi^{1}$ rotameric configuration of the catalytically active state at high pH (Fig. 3, upper right panel). Supporting our model of dual interactions of T264 and H260 with the catalytic water molecule, a sidechain-flipped configuration of $\mathrm{H} 260$ captured under the acidic condition in our crystal structure of the $\mathrm{HK} 853^{\mathrm{CP}}-\mathrm{BeF}_{3}{ }^{-}$RR468 complex would result in a loss of the critical hydrogen bond between $\mathrm{H} 260$ and the catalytic water molecule and the perturbation of the chemical shift of the K105-interacting fluorine $F_{2}$ to the catalytically inactive state (Figs. 1c, 3, lower right panel). Further elimination of the hydroxyl group in the T264A HK853 mutant would result in a complete loss of the catalytic water molecule (Fig. 3, lower left panel), returning the chemical environment of the $F_{2}$ fluorine atom in the T264A HK853- $\mathrm{BeF}_{3}{ }^{-}$-RR468 complex to that of the substrate state of $\mathrm{BeF}_{3}{ }^{-}$-RR468 (Fig. 3, upper left panel). A similar water molecule has been previously hypothesized to interact with a conserved glutamine residue of the DxxQ motif in the nitrogen sensor NarX of the HisKA_3 family based on mutagenesis studies, though the phospho-accepting histidine residue was not thought to be involved in the water interaction ${ }^{8}$.

In order to probe the chemical environment of the HK853 $3^{\mathrm{cP}}$ T264A mutant and gain further structural support for our proposed model of dual interactions of HK853 ${ }^{\mathrm{cp}} \mathrm{T} 264$ and H260 with the catalytic water molecule, we crystallized the T264A $\mathrm{HK} 853^{\mathrm{CP}}-\mathrm{BeF}_{3}{ }^{-}-\mathrm{RR} 468$ complex under the same condition as the wild-type complex at $\mathrm{pH}$ 5.0, and determined the structure at 3.6 $\AA$ resolution (Table 1, a portion of the electron density map is shown in Supplementary Fig. 9). Consistent with our proposed model (Fig. 3), we found that the structure of the T264A mutant complex indeed captured the same catalytically inactive conformation as the wild-type complex at $\mathrm{pH}$ 5.0, with $\mathrm{H} 260$ adopting a gauche- $\chi^{1}$ rotameric conformation (Fig. 4a, Supplementary Fig. 9). The overall backbone rmsd between the two structures is $0.32 \AA$, confirming that the T264A mutation did not affect the binding interface or the stability of the protein complex. However, with the loss of the hydroxyl sidechain in the T264A mutation and with H260 swung away from the active site in a catalytically inactive, gauche- $\chi^{1}$ rotameric conformation (Fig. $4 \mathrm{~b}$ ), none of the water bridging functional groups is retained in the mutated $\mathrm{HK} 853^{\mathrm{CP}}-\mathrm{BeF}_{3}{ }^{-}-\mathrm{RR} 468$ complex, leaving the $\mathrm{F}_{2}$ fluorine 
a

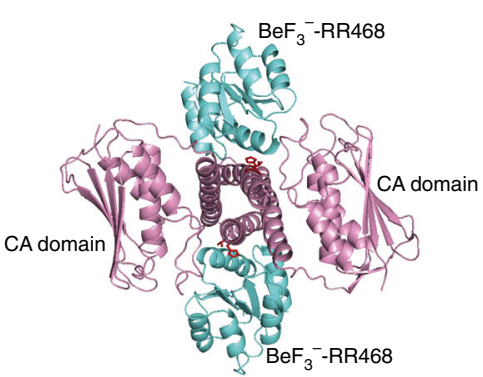

C

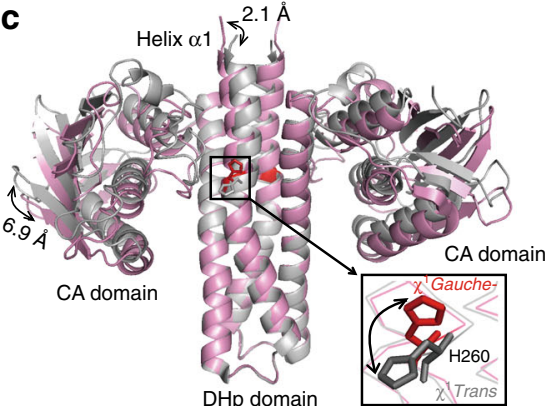

e

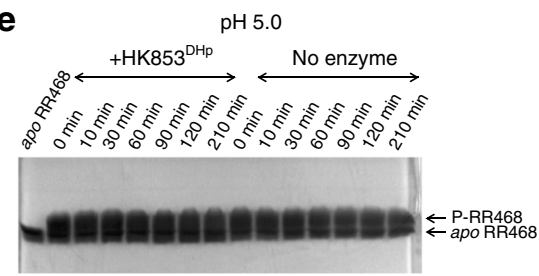

b

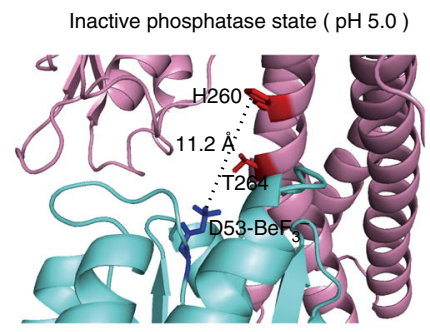

d

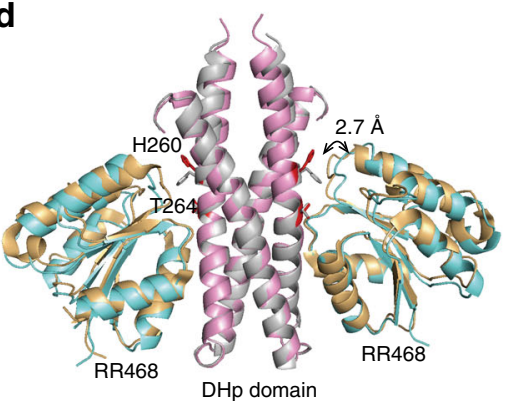

f

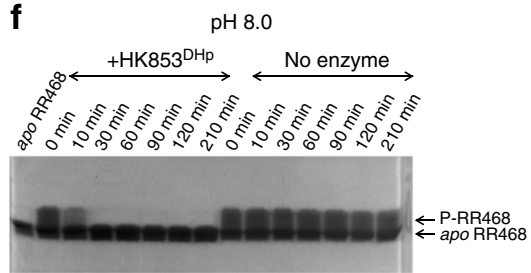

Fig. 2 Crystal structure of the $\mathrm{HK}_{85} 3^{\mathrm{CP}}-\mathrm{BeF}_{3}{ }^{-}-\mathrm{RR} 468$ complex at low pH reveals a catalytically inactive state. a The structure of the $\mathrm{HK} 853^{\mathrm{CP}}-\mathrm{BeF}_{3}{ }^{-}-\mathrm{RR}_{4} 68$ complex at pH 5.0. The $\mathrm{BeF}_{3}{ }^{-}$-RR468 substrate is shown in cyan, the HK853 ${ }^{\mathrm{cP}}$ dimer in pink, and the sidechains of $\mathrm{H} 260$ and $\mathrm{T} 264$ of $\mathrm{HK} 853^{\mathrm{CP}}$ in red. $\mathbf{b}$ Structural details of the inactive state of the $\mathrm{HK} 853^{\mathrm{CP}}-\mathrm{BeF}_{3}{ }^{-}$-RR468 complex at $\mathrm{pH}$ 5.0. The $\mathrm{BeF}_{3}{ }^{-}$-D53 residue of RR468 is shown in blue, and the distance between the $\varepsilon-\mathrm{N}$ atom of $\mathrm{H} 260$ and the Be atom is labeled. $\mathbf{c ~ A ~ p H - g a t e d ~ c o n f o r m a t i o n a l ~ s w i t c h ~ o f ~} \mathrm{HK} 853^{\mathrm{cp}}$. Superimposed structures of the inactive state (pink, with the sidechain of $\mathrm{H} 260$ in red; this structure) and active state (gray, PDB 3DGE) of the $\mathrm{HK} 83^{\mathrm{CP}}-\mathrm{BeF}_{3}{ }^{-}-\mathrm{RR} 468$ complex demonstrate the sidechain flipping of $\mathrm{H} 260$ and the movements of the CA domain and the N-terminal $\alpha 1$-helix of the DHp domain. The distances between $\mathrm{C} \alpha$ atoms of C359 of the CA domain and between $\mathrm{C} \alpha$ atoms of R246 of the DHp domain in the two structures are labeled. For visual clarity, RR468 is not displayed. The $\chi^{1}$ rotameric states of $\mathrm{H} 260$ in the two structures are labeled. $\mathbf{d}$ Structural movement of RR468 in the $\mathrm{HK} 853^{\mathrm{CP}}-\mathrm{BeF}_{3}{ }^{-}-\mathrm{RR}_{4} 48$ complex between the inactive state (with RR468 shown in cyan) and active state (with RR468 shown in orange). The distance between $\mathrm{C} \alpha$ atoms of G87 in the two structures is labeled. For visual clarity, the CA domains are not displayed. Structural overlays in $\mathbf{c}, \mathbf{d}$ are generated by superimposing the $\alpha 2$-helix and the $\mathrm{C}$-terminal half of the $\alpha 1$-helix (residues of 266-317) of the DHp domain of HK853 ${ }^{\mathrm{CP}}$. e, $\mathbf{f}$ show the differential phosphatase activities of HK853 ${ }^{\mathrm{DHp}}$ detected by the Phos-tag gel shift assay at $\mathrm{pH} 5.0$ and $\mathrm{pH} 8.0$, respectively

in a chemical environment identical to that in apo $\mathrm{BeF}_{3}{ }^{-}$- $\mathrm{RR} 468$. Taken together, these observations provide strong support for the dual engagement model of the catalytic water molecule by both H260 and T264 of HK853 (Fig. 3).

pH-regulated phosphatase activity in the EnvZ-OmpR TCS. In Salmonella, expression of the type III secretion system genes encoded by the Salmonella Pathogenicity Island (SPI) 2 is required for systemic infection in mice and is regulated by the $s s r A$-ssr $B$ genes $^{20}$ (Fig. 5a). Active transcription of the $s s r A-s s r B$ genes occurs after Salmonella enters host cells in an acidified vacuole, accompanied by the accumulation of phospho-OmpR, the response regulator of the classical EnvZ/OmpR twocomponent system ${ }^{21}$. EnvZ belongs to the same HisKA subfamily as HK853 and shares a similar bifunctional motif (Fig. 5b). It has recently been reported that intravacuolar Salmonella is acidified in response to external acid stress ${ }^{4}$, suggesting that the cytoplasmic $\mathrm{pH}$ could be an important regulator of the bifunctional histidine kinase EnvZ that leads to the accumulation of phospho-OmpR.
In order to test this scenario, we first evaluated the $\mathrm{pH}$ dependent phosphatase activity of EnvZ by the Phos-tag assay using the purified cytoplasmic portion of EnvZ (EnvZ ${ }^{\mathrm{cP}}$ ) and the receiver domain of the response regulator OmpR (OmpRr). Similar to HK853, we found that EnvZ $Z^{\mathrm{cp}}$ is a less efficient phosphatase under acidic conditions than under neutral $\mathrm{pH}$ conditions (Fig.5c; uncropped image shown in Supplementary Fig. 10). Based on these in vitro observations, we went on to examine whether the $\mathrm{pH}$-regulated phosphatase activity of the bifunctional histidine kinase EnvZ is responsible for the accumulation of the phospho-OmpR and transcriptional activation of $s s r A$ and $s s r B$ in vivo using a quantitative real-time PCR assay. As OmpR is the specific response regulator of EnvZ, the catalytically inactive H243A EnvZ mutant does not generate phospho-OmpR; therefore the background transcriptional levels of $s s r A$ and $s s r B$ were normalized to one in the envZ$H 243$ A mutant strain at $\mathrm{pH} 5.0$ and 7.0, respectively, for comparison with the wild-type strain and the envZ-T247A mutant strain under different $\mathrm{pH}$ conditions. In a neutral $\mathrm{pH}$ environment ( $\mathrm{pH} 7.0$ ), we observed a modest enhancement of the expression of both $s s r A$ and $s s r B$ due to the presence of 

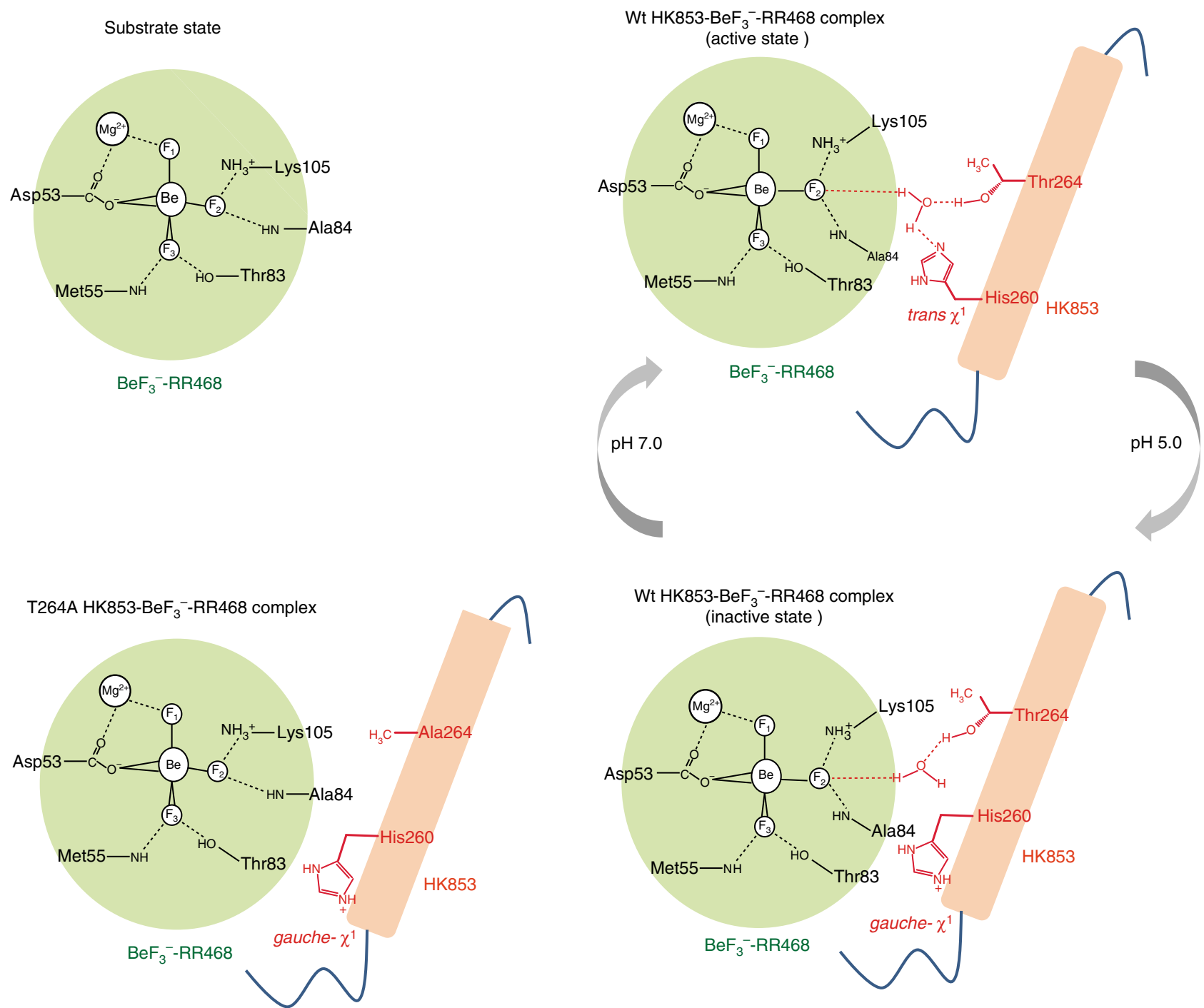

Fig. 3 Proposed role of the conserved HxxxT motif in the pH-gated phosphatase activity of HisKA. Residues in the active site of RR468 and HK853 are shown in black and red, respectively. In the proposed model, the substrate state (upper left) defines the apo state fluorine chemical shifts, which reflects a lack of specific interactions of the $F_{2}$ fluorine atom with HK853. In the catalytically active state ( $F^{c a}$ state, upper right), the $F_{2}$ fluorine atom (mimicking an oxygen atom of the phosphate group) forms a hydrogen bond with the catalytic water molecule, which is activated by T264 and H260 of HK853, with

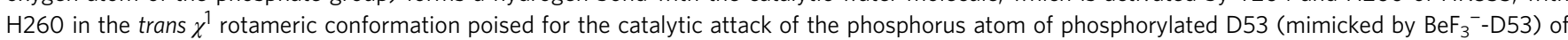
RR468. Under acidic conditions, the protonated H260 swings away from the catalytic water molecule, leaving HK853 T264 as the only remaining residue interacting with the catalytic water molecule and the $\mathrm{F}_{2}$ fluorine atom of $\mathrm{BeF}_{3}{ }^{-}-\mathrm{RR} 468$, and reflecting a catalytically inactive state of $\mathrm{HK} 853$ ( $\mathrm{F}^{\mathrm{ci}}$, lower right). The $\mathrm{F}_{2}$ atom of $\mathrm{BeF}_{3}{ }^{-}-\mathrm{D} 53$ in the T264A complex shows a similar chemical environment to the $\mathrm{F}_{2}{ }^{\text {apo }}$ state despite the formation of the T264A $\mathrm{HK} 853-\mathrm{BeF}_{3}{ }^{-}-\mathrm{RR} 468$ complex, consistent with the loss of the $\mathrm{F}_{2}$-interacting catalytic water molecule due to the change of $\mathrm{H} 260 \chi^{1}$ rotameric conformation to gauche- and the elimination of the side chain hydroxyl group from the T264A mutation (lower left)

phospho-OmpR generated by the kinase activity in the wild-type bifunctional EnvZ enzyme (Fig. 5d); however, the enhancements of mRNA levels of $s s r A$ and $s s r B$ under neutral $\mathrm{pH}$ were significantly lower than those under the acidic environment ( $\mathrm{pH}$ 5.0). Such an in vivo observation is consistent with our in vitro discovery that the phosphatase activity of bifunctional histidine kinases is diminished by a $\mathrm{pH}$-gated conformational switch, resulting in the accumulation of a higher level of phospho-OmpR in the acidic environment. In contrast, although the mRNA levels of $s s r A$ and $s s r B$ in the envZ-T247A mutant strain deficient in the phosphatase activity are enhanced in comparison to those in the wild-type EnvZ strain, the variation in the mRNA levels of $s s r A$ and $s s r B$ under different $\mathrm{pH}$ environments is statistically insignificant, confirming that the observed changes in the mRNA levels of $s s r A$ and $s s r B$ under the control of wild-type EnvZ is directly caused by the differential phosphatase activity mediated by a $\mathrm{pH}$-gated conformational switch, but not other factors.

Taken together, our data suggest that a $\mathrm{pH}$-gated inactivation of the phosphatase activity of EnvZ directly regulates the accumulation of the phosphorylated response regulator OmpR and expression of SPI2-encoded genes of the two-component system ( $s s r A$ and $s s r B$ ) and the type III secretion system for enhanced bacterial infectivity. Such a model is further supported by the Salmonella host invasion experiment: ablation of the kinase activity in the H243A EnvZ mutant eliminated the production of phospho-OmpR and profoundly diminished the macrophage infectivity of the mutant bacterium compared to the wild-type strain, whereas eliminating the phosphatase activity in the T247A EnvZ mutant resulted in an enhanced macrophage 
a

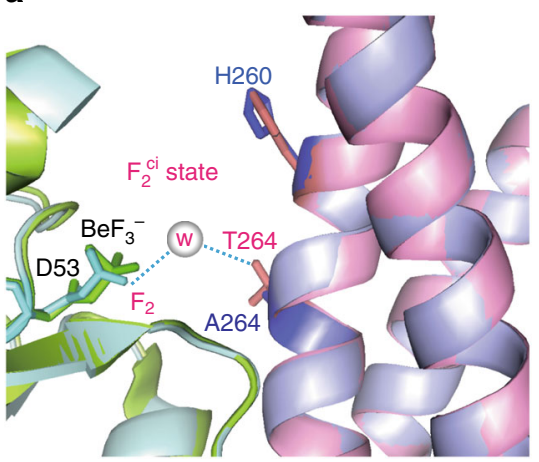

b

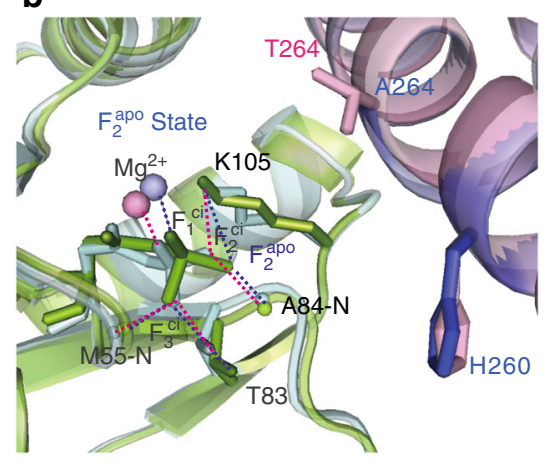

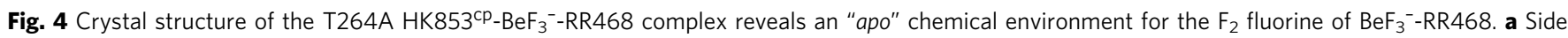
view of the active site of the T264A HK853 ${ }^{\mathrm{CP}}-\mathrm{BeF}_{3}{ }^{-}-\mathrm{RR} 468$ complex overlaid with that of the wild-type complex crystallized at $\mathrm{pH} 5.0$. In the T264A complex, the $\mathrm{BeF}_{3}{ }^{-}-\mathrm{RR} 468$ substrate is shown in green and the T264A HK853 ${ }^{\mathrm{CP}}$ in pale purple, with the sidechains of $\mathrm{H} 260$ and $\mathrm{A} 264$ of $\mathrm{T} 264 \mathrm{~A} H K 853^{\mathrm{CP}}$ in the stick model. In the wild-type complex, the $\mathrm{BeF}_{3}{ }^{-}-\mathrm{RR} 468$ substrate is shown in cyan and $\mathrm{HK}^{2} 53^{\mathrm{CP}}$ in pink, with the sidechains of $\mathrm{H} 260$ and T264 of $\mathrm{HK} 853^{\mathrm{CP}}$ shown in the stick model. The interaction between the $\mathrm{F}_{2}$ atom of $\mathrm{BeF}_{3}{ }^{-}$-D53 and the hydroxyl group of T264, bridged by the proposed catalytic water molecule (shown in the sphere model) in the wild-type complex at low pH generates the $\mathrm{F}_{2}{ }^{\mathrm{ci}}$ state. The loss of the catalytic water and its bridged interactions between the $\mathrm{F}_{2}$ atom of $\mathrm{BeF}_{3}{ }^{-}-\mathrm{RR} 468$ and $\mathrm{T} 264$ and $\mathrm{H} 260$ in the T264A HK853 ${ }^{\mathrm{CP}}$ mutant generates a chemical environment similar to the

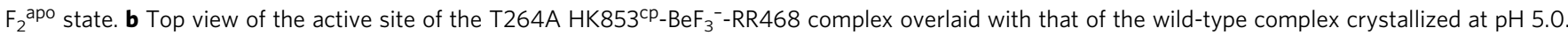
Interactions of the fluorine atoms in $\mathbf{a}$ and $\mathbf{b}$ are indicated by dashed lines

infectivity due to the accumulation of phospho-OmpR (Fig. 5e, Supplementary Fig. 11).

\section{Discussion}

The bifunctional histidine kinases of bacterial two-component systems possess both phosphoryl transfer activity (kinase) and phosphoryl removal activity (phosphatase) toward their cognate response regulators. Despite the essential roles of these enzymes in regulating bacterial pathogenesis in response to the change of the host environment during infection, the molecular mechanisms that regulate the opposing kinase and phosphatase activities are only starting to be elucidated. In the ChpT-CtrA two-component system of Brucella abortus ${ }^{22}$, it was suggested that the quaternary structure of the $\mathrm{ChpT}_{2}-\mathrm{CtrA}_{2}$ complex enforces an asymmetric mechanism of phosphoryl transfer between ChpT and $\mathrm{CtrA}$, and only one of the two asymmetric binding modes is competent in the phosphotransfer activity of ChpT. However, the molecular mechanism regulating the phosphatase activity in this system remains entirely unclear. Recently, the regulatory mechanism of the bifunctional histidine kinase CckA from Caulobacter crescentus was revealed ${ }^{23}$. In this case, binding of the second messenger cyclic di-guanosine monophosphate (c-diGMP) suppresses the default kinase activity of CckA through noncovalent cross-linking of the catalytic domain with the dimerization histidine phosphotransfer domain. Our study represents a complementary regulatory mechanism of bifunctional histidine kinases that inactivates the phosphatase activity through a pH-gated conformational switch (Fig. 3). It is important to note that the conformation-dependent inactivation process occurs on the slow exchange time scale for NMR measurements ( $\mu$ s-to-ms) and yields two sets of signals corresponding to the conformations of the active and inactivate states between pH 5.2 and 6.5 (Fig. 1d). Such a mechanism is distinct from the general reduction of the catalytic activity due to protonation of the catalytic base, where the fast protonationdeprotonation process yields a single set of population-weighted signals as observed in many enzymes ${ }^{24}$. Interestingly, although the catalytically inactive conformation reported here has not been previously captured in wild-type enzymes, it has been observed in a "rewired" HK853 ${ }^{\mathrm{cP}}{ }^{*}-\mathrm{RR} 468^{*}$ complex, in which the interface residues of HK853 and RR468 from Thermotoga maritima were mutated to match those of the two-component system in Escherichia coli, PhoR and PhoB, respectively ${ }^{25}$. As these mutations also perturbed the arrangement and relative orientation of the histidine kinase with respect to the response regulator, the critical factor that trapped the chimeric complex into the inactive state has not been isolated. In comparison, by combining solution NMR and crystallographic studies of the wild-type HK853-RR468 complex, we have been able to establish the critical role of $\mathrm{pH}$ in regulating the conformation-dependent phosphatase activity of the HisKA family of bifunctional histidine kinases. Whether the inactive conformation of the wild-type HK853RR468 complex observed at low $\mathrm{pH}$ could resemble snapshots of transient intermediates of the phosphotransfer reaction as previously suggested for the rewired complex ${ }^{25}$ remains to be investigated.

In summary, our combined solution NMR and crystallographic studies have revealed a $\mathrm{pH}$-gated conformational switch that modulates the phosphatase activity of the largest family of bifunctional histidine kinases. Given the broad conservation of residues involved in this conformation switch, such a regulatory mechanism likely reflects a general $\mathrm{pH}$-response mechanism for the vast majority of the two-component signal transduction systems that function in bacteria, plants and fungi.

\section{Methods}

Protein preparation and purification. Plasmids encoding $\mathrm{HK} 853^{\mathrm{cp}}$ (residues 232-489), RR468, EnvZ ${ }^{\mathrm{cp}}$ (residues 215-450), OmpR receiver domain (OmpRr; residues 1-125) and $\mathrm{His}_{6}$-MBP-HK853 ${ }^{\mathrm{DHP}}$ (residues 250-315) were transformed into E. coli BL21(DE3) Gold cells (Novagen). For unlabeled protein, the transformed bacterial cells were cultivated in the LB medium containing either ampicillin $\left(100 \mu \mathrm{g} \mathrm{mL} L^{-1}\right)$ or kanamycin $\left(50 \mu \mathrm{g} \mathrm{m}^{-1}\right)$ at $37^{\circ} \mathrm{C}$, and then induced with IPTG (isopropyl $\beta$-d-1-thiogalactopyranoside; $1 \mathrm{mM}$ ) at $20^{\circ} \mathrm{C}$ for $20 \mathrm{~h}$ before collecting. ${ }^{15} \mathrm{~N}$-labeling of RR468 was achieved by growing cells in the M9 minimal medium containing ${ }^{15} \mathrm{~N} \mathrm{NH}_{4} \mathrm{Cl}$ as the sole nitrogen source.

$\mathrm{HK} 853^{\mathrm{cP}}$ was purified by ammonium sulfate precipitation $(40 \% \mathrm{w} / \mathrm{v})$ followed by anion-exchange and size-exclusion chromatography. RR468 was purified by incubation at $60^{\circ} \mathrm{C}$ followed by anion-exchange and size-exclusion chromatography ${ }^{5,26}$. Similar protocols were used to purify EnvZ ${ }^{\mathrm{cp}}$ and OmpRr. The H260A and T264A point mutants of HK853 ${ }^{\mathrm{DHp}}$ were generated by point mutagenesis (primers summarized in Supplementary Table 2). Wild-type and mutant $\mathrm{HK} 853^{\mathrm{DHp}}$ were expressed as $\mathrm{His}_{6}$-MBP fusion proteins. Each fusion protein was purified by using the amylose affinity chromatography and eluted with the buffer containing $20 \mathrm{mM}$ Tris (pH 7.6), $20 \mathrm{mM}$ maltose and $300 \mathrm{mM} \mathrm{NaCl}$. The $\mathrm{His}_{6}$-MBP tag was cleaved by the tobacco etch virus (TEV) protease digestion and removed by passing the cleavage sample through an amylose column in a buffer 
a

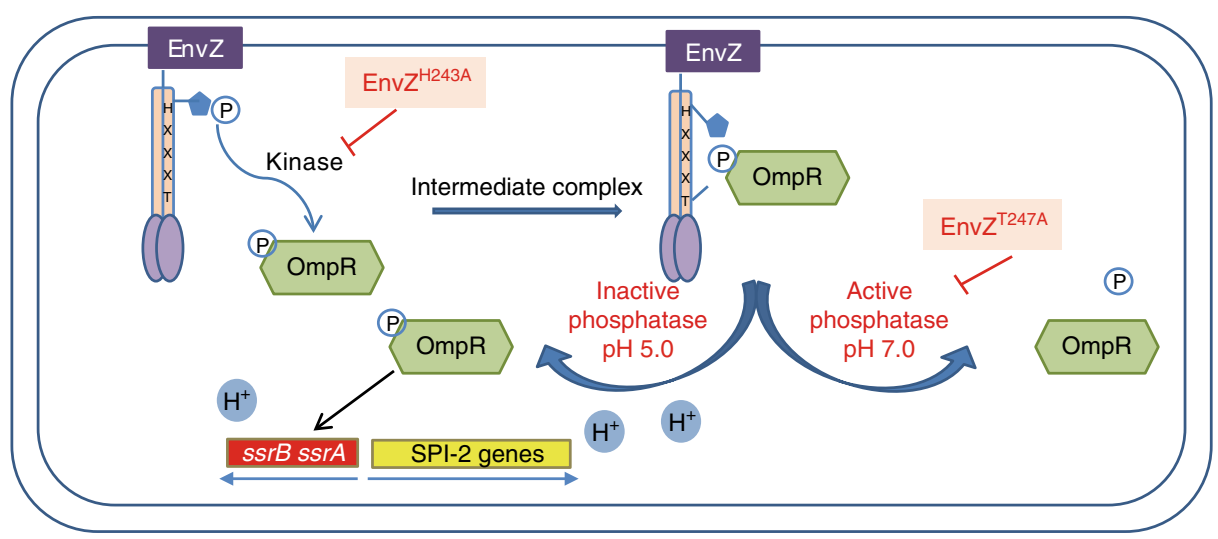

b

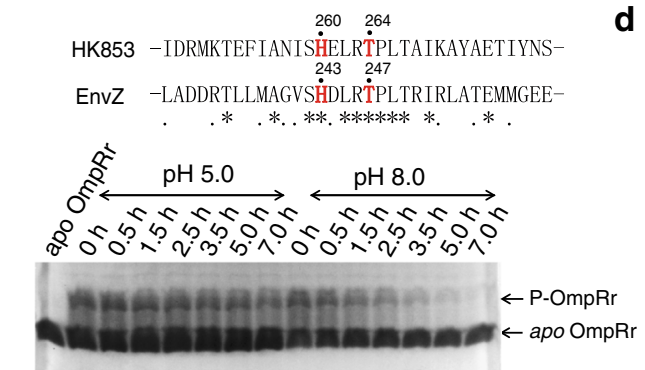

e

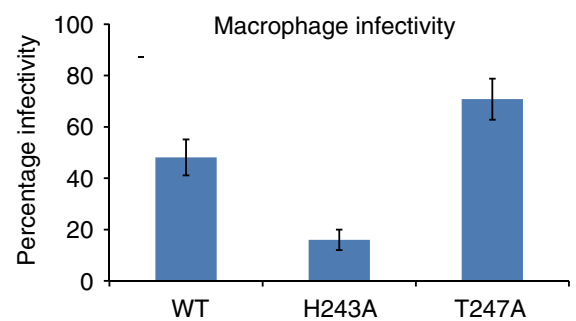

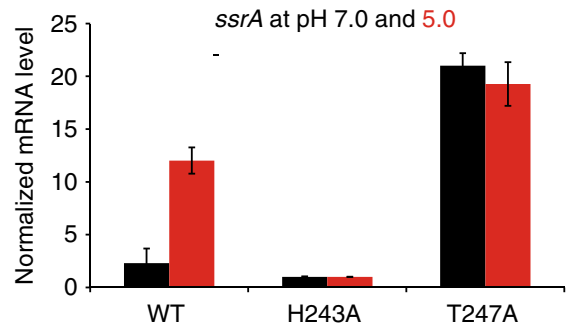

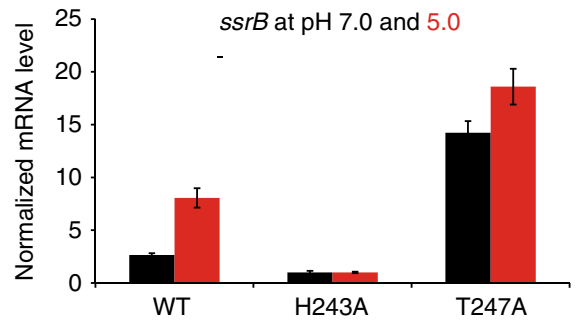

Fig. $5 \mathrm{pH}$-gated regulation of the phosphatase activity of the bifunctional histidine kinase EnvZ in Salmonella. a The schematic illustration depicting the different functions of EnvZ at different pHs during host cell invasion. The H243A and T247A point mutations abolish the kinase and phosphatase activities of EnvZ, respectively. $\mathbf{b}$ Sequence alignment of the $\alpha_{1}$ helix in EnvZ and HK853 from the same HisKA subfamily, with the HxxxT motif highlighted in red. Identical and conserved residues are indicated below the sequence alignment. $\mathbf{c}$ Differential phosphatase activities of Env $Z^{\mathrm{CP}}$ detected by the Phos-tag gel shift assay at pH 5.0 and 8.0, respectively. $\mathbf{d}$ Detection of the expression of ssrA and ssrB genes by real-time fluorescence quantitative PCR at different pHs. The expression levels at pH 7.0 and 5.0 were shown in black and red, respectively. In each group, the expression level of the $\mathrm{H} 243 \mathrm{~A}$ mutant is normalized to 1.0 for comparison. e Macrophage infectivity of S. typhimurium LT2 and mutants. The infectivity was defined as the percentage of infected host cells. Error bars in $\mathbf{d}$ and $\mathbf{e}$ represent the standard deviation from quadruplet measurements

containing $20 \mathrm{mM}$ Tris (pH 7.6) and $300 \mathrm{mM} \mathrm{NaCl}$. The target $\mathrm{HK} 853^{\text {DHp }}$ bound weakly to the $\mathrm{Ni}^{2+}$-NTA column and was separated from the His ${ }_{6}$-tagged TEV by elution in a buffer containing $50 \mathrm{mM}$ phosphate (pH 8.0), $300 \mathrm{mM} \mathrm{NaCl}$, and $30 \mathrm{mM}$ imidazole. All protein samples were further purified to homogeneity using size-exclusion chromatography.

NMR experiments. Protein samples were dissolved in the NMR buffer containing $20 \mathrm{mM}$ HEPES (pH 5.0-7.0), $50 \mathrm{mM} \mathrm{KCl}$, and $10 \mathrm{mM} \mathrm{MgCl}_{2}$, with various levels of $\mathrm{D}_{2} \mathrm{O}$. For NMR samples without $\mathrm{D}_{2} \mathrm{O}$, a hermetical tube containing $\mathrm{D}_{2} \mathrm{O}$ was inserted into the NMR tube for the field lock.

$\mathrm{BeF}_{3}{ }^{-}-\mathrm{RR} 468$ was generated by adding $5 \mathrm{mM} \mathrm{BeCl}{ }_{2}$ and $50 \mathrm{mM} \mathrm{NaF}$ to the RR468 solution, which was incubated at room temperature for $30 \mathrm{~min}$. The protein complexes were generated by adding $\mathrm{HK} 853^{\mathrm{DHp}}$ or mutants at a concentration 10 fold higher than the substrate $\mathrm{BeF}_{3}{ }^{-}$-RR468.

The ${ }^{19} \mathrm{~F}$ NMR experiments ${ }^{13,14}$ were performed on a $600 \mathrm{MHz}$ Bruker spectrometer (equipped with a $5 \mathrm{~mm} C P Q C I{ }^{1} \mathrm{H} /{ }^{19} \mathrm{~F}-{ }^{-13} \mathrm{C} /{ }^{15} \mathrm{~N} / \mathrm{D}$ Z-GRD cryoprobe) at $298 \mathrm{~K}$, and the chemical shifts were referenced to $\mathrm{CFCl}_{3}$ (the free fluorine signal $\mathrm{F}^{-}$at -119.0 p.p.m.).

The pulse sequence for the $\mathrm{H} 3(\mathrm{~N}) \mathrm{F}$ experiment was adapted from the $\mathrm{H} 3(\mathrm{~N}) \mathrm{P}$ experiment originally designed to analyze the ${ }^{3 h} J_{N P}$ coupling ${ }^{27}$. For the ${ }^{19} \mathrm{~F}$ dimension, the carrier position was set to -156.0 p.p.m. The evolution decay in the ${ }^{15} \mathrm{~N}$ dimension was set to $35.0 \mathrm{~ms}$. The chemical shift of lysine sidechain $\mathrm{NH}_{3}$ was assigned by $2 \mathrm{D}^{1} \mathrm{H}^{15}{ }^{15} \mathrm{~N}$ HISQC, $3 \mathrm{D}(\mathrm{H}) \mathrm{CCENH} 3$, and $3 \mathrm{D}(\mathrm{H}) \mathrm{CCNH}$ experiments ${ }^{28,29}$.
NMR data were processed with NMRpipe ${ }^{30}$ and analyzed by Topspin 3.2 .

Protein crystallization and structural analysis. The protein complex $(10 \mathrm{mg} \mathrm{mL}$ ${ }^{-1} \mathrm{HK} 853^{\mathrm{cP}}$ and $7.5 \mathrm{mg} \mathrm{mL}^{-1} \mathrm{RR} 468$ ) was dissolved in a buffer containing $10 \mathrm{mM}$ Tris (pH 8.0), $7 \mathrm{mM} \mathrm{MgCl} 2,5 \mathrm{mM} \mathrm{BeCl}_{2}, 30 \mathrm{mM} \mathrm{NaF}$, and $4 \mathrm{mM} \mathrm{AMPPNP}^{5}$. Crystallization of the wild-type complex was carried out by using the sitting drop vapor diffusion method at $20^{\circ} \mathrm{C}$ in drops containing equal volumes of the protein complex and mother liquor consisting of $0.1 \mathrm{M}$ citric acid ( $\mathrm{pH} 4.0), 0.8 \mathrm{M}$ ammonium sulfate, with the final $\mathrm{pH}$ adjusted to 5.0. For the T264A mutant complex, the mother liquor contained $0.1 \mathrm{M}$ citric acid (pH 5.0), $0.208 \mathrm{M}$ ammonium sulfate, and crystallization was carried out at $15^{\circ} \mathrm{C}$ by using the hanging drop vapor diffusion method. The crystals were cryoprotected by a solution containing $50 \%$ mother liquor $(\mathrm{v} / \mathrm{v})$ and $50 \%$ glycerol $(\mathrm{v} / \mathrm{v})$ for the wildtype complex and $50 \%$ mother liquor $(\mathrm{v} / \mathrm{v})$ and $50 \%$ sucrose $(\mathrm{v} / \mathrm{v})$ for the T264A mutant complex, respectively. The data were collected at the SER-CAT 22-ID and 22-BM lines at Argonne National Laboratory for the wild-type and T264A mutant complexes, respectively; diffraction data were processed with XDS ${ }^{31}$ or HKL2000 32 and truncated and scaled with the University of California Los Angeles anisotropy diffraction server ${ }^{33,34}$. The previously reported structure of the HK853 ${ }^{\mathrm{CP}}$-RR468 complex crystallized at pH 5.6 (PDB: 3DGE) ${ }^{5}$ was used as the search model for molecular replacement by using the PHENIX suite ${ }^{35}$. The structural model was iteratively built by $\mathrm{COOT}^{36}$ and refined by PHENIX. In the crystal structure of the wild-type HK853 $3^{\mathrm{CP}}$-RR468 complex, additional electron density was observed near 
one of the two $\mathrm{H} 260$ residues, which was interpreted as a glycerol molecule from the cryo-protectant.

Dephosphorylation assay in vitro. Samples of RR468 and OmpRr were phosphorylated at a protein concentration of $4 \mathrm{mg} \mathrm{mL}^{-1}$ in the presence of $12 \mathrm{mM}$ acetylphosphate at $25^{\circ} \mathrm{C}$ for $1 \mathrm{~h}$. The free phosphate was removed by using a GE desalting column. Samples of phosphorylated RR468 and phosphorylated OmpRr were exchanged into desired reaction buffers (20 mM Tris, $10 \mathrm{mM} \mathrm{MgCl}_{2}, 100 \mathrm{mM}$ $\mathrm{NaCl}$ at $\mathrm{pH} 8.0$; and $20 \mathrm{mM} \mathrm{NaAc}, 10 \mathrm{mM} \mathrm{MgCl}_{2}, 100 \mathrm{mM} \mathrm{NaCl}$ at $\mathrm{pH} 5.0$ ), flashfrozen, and stored at $-80^{\circ} \mathrm{C}$.

A reaction mixture of phospho-RR468 and HK853 ${ }^{\mathrm{DHp}}$ or mutant enzymes at a molar ratio of $6: 1$ was incubated at $4{ }^{\circ} \mathrm{C}$ for the detection of the phosphatase activity by the native polyacrylamide gel electrophoresis (PAGE) assay, whereas a molar ratio of 13.8:1 was used for the Phos-tag gel assay. The same protocol was used to assay the phosphatase activity of the EnvZ ${ }^{\mathrm{cp}}$ and mutants towards phospho-OmpRr. Samples at different time points were taken by flash-freezing at $-80^{\circ} \mathrm{C}$. For the native-PAGE detection, gels were run on ice at $90 \mathrm{~V}$ for $5 \mathrm{~h}$. The Phos-tag gel was made by adding $75 \mathrm{uM}$ Phos-tag acrylamide and $150 \mu \mathrm{M} \mathrm{MnCl}_{2}$ into the SDS-PAGE ${ }^{37}$. All the gels used for assays were stained by Coomassie blue.

Construction of Salmonella EnvZ mutant strains. S. typhimurium strains containing the envZ H243A or T247A mutations were constructed from the LT2 strain $^{38,39}$. Briefly, the envZ gene in LT2 strain was first replaced by a kanamycin resistance gene (kan) to obtain the envZ::kan strain using the pKD46mediated gene disruption system ${ }^{40}$. The kanamycin resistance gene in the envZ: kan strain was subsequently replaced with the H243A or T247A mutant of the env $Z$ gene using a homologous recombination method as previously described ${ }^{41}$ Detailed procedures are described in Supplementary Methods.

RNA extraction and qRT-PCR. Cells of $S$. typhimurium strains were grown in a modified N-minimal medium (MgM) buffered with $20 \mathrm{mM}$ Tris ( $\mathrm{pH} 7.0$ ) or $20 \mathrm{mM}$ MES (pH 5.0) containing $7.5 \mathrm{mM}\left(\mathrm{NH}_{4}\right)_{2} \mathrm{SO}_{4}, 5 \mathrm{mM} \mathrm{KCl}, 0.5 \mathrm{mM} \mathrm{K}_{2} \mathrm{SO}_{4}$, $1 \mathrm{mM} \mathrm{KH}_{2} \mathrm{PO}_{4}, 10 \mathrm{mM} \mathrm{MgCl}_{2}, 2 \mathrm{mM}$ glucose, and $0.1 \%$ Casamino acids ${ }^{4}$. When $\mathrm{OD}_{600}$ reached 0.5 , cells were collected by centrifugation. Total RNA of each collection was extracted with the TRIzol reagent (Invitrogen) according to the manufacturer's protocol. After treatment with RNase-free DNase I (Promega), $2 \mu \mathrm{g}$ of each RNA sample was used as the template to obtain the cDNA by RNA PCR kit (AMV) ver. 3.0 (TaKaRa). Real-time PCR was performed using Universal SYBR Green Supermix Reagent (Bio-Rad). Results were normalized to the 16S rRNA level. For each strain, wild-type or mutants, all tests were repeated four times independently. Mean values and standard deviations (SD) of these experiments were calculated. Detailed information of PCR primers is listed in Supplementary Information.

Immunofluorescence detection of macrophage infection. S. typhimurium LT2 cells were grown in the $\mathrm{LB}$ medium overnight at $37^{\circ} \mathrm{C}$ until $\mathrm{OD}_{600}$ reached 1.0. The RAW 264.7 host cells (FineTest, Wuhan) were cultured at $37^{\circ} \mathrm{C}$ and then infected by LT2 at a multiplicity of infection of 100 for $30 \mathrm{~min}$ followed by PBS washes. The host cells were further incubated for $4 \mathrm{~h}$, then fixed with $4 \%$ formaldehyde, permeabilized in $0.2 \%$ Triton X-100, and treated with 5\% FBS before antibody incubation. LT2 cells were incubated with anti-Salmonella Csa-1 primary antibody (KPL) and DyLight 549-conjugated AffiniPure rabbit anti-sheep IgG (EarthOx) second antibody at $4{ }^{\circ} \mathrm{C}$ overnight. The host cells were visualized by FITCconjugated phalloidin (Sigma-Aldrich) that stains actin filaments. The excitation wavelengths of the two different fluorescent dyes were 495 and $552 \mathrm{~nm}$, respectively. The final images were visualized and processed by confocal microscopy.

Data availability. The coordinates and structure factors for the X-ray structures of the wild-type $\mathrm{HK} 853^{\mathrm{CP}}-\mathrm{BeF}_{3}{ }^{-}$-RR468 complex and the T264A mutant complex at $\mathrm{pH} 5.0$ have been deposited to the Protein Data Bank (PDB) with accession codes 5 UHT and 6AZR, respectively. Other data are available from the corresponding author upon request.

Received: 17 January 2017 Accepted: 20 November 2017 Published online: 13 December 2017

\section{References}

1. Stock, A. M., Robinson, V. L. \& Goudreau, P. N. Two-component signal transduction. Annu. Rev. Biochem. 69, 183-1215 (2000).

2. Gotoh, Y. et al. Two-component signal transduction as potential drug targets in pathogenic bacteria. Curr. Opin. Microbiol. 13, 232-239 (2010).

3. Capra, E. J. \& Laub, M. T. Evolution of two-component signal transduction systems. Annu. Rev. Microbiol. 66, 325-347 (2012).
4. Chakraborty, S., Mizusaki, H. \& Kenney, L. J. A FRET-based DNA biosensor tracks OmpR-dependent acidification of Salmonella during macrophage infection. PLoS Biol. 13, e1002116 (2015).

5. Casino, P., Rubio, V. \& Marina, A. Structural insight into partner specificity and phosphoryl transfer in two-component signal transduction. Cell 139, 325-336 (2009).

6. Szurmant, H. \& Hoch, J. A. Statistical analyses of protein sequence alignments identify structures and mechanisms in signal activation of sensor histidine kinases. Mol. Microbiol. 87, 707-712 (2013).

7. Casino, P., Rubio, V. \& Marina, A. The mechanism of signal transduction by two-component systems. Curr. Opin. Struc. Biol. 20, 763-7771 (2010).

8. Huynh, T. N., Noriega, C. E. \& Stewart, V. Conserved mechanism for sensor phosphatase control of two-component signaling revealed in the nitrate sensor NarX. Proc. Natl Acad. Sci. USA 107, 21140-21145 (2010).

9. Willett, J. W. \& Kirby, J. R. Genetic and biochemical dissection of a HisKA domain identifies residues required exclusively for kinase and phosphatase activities. PLoS Genet. 8, e1003084 (2012).

10. Kern, D. et al. Structure of a transiently phosphorylated switch in bacterial signal transduction. Nature 402, 894-898 (1999).

11. Hastings, C. A. et al. High-resolution solution structure of the beryllofluorideactivated NtrC receiver domain. Biochemistry 42, 9081-9090 (2003).

12. Wemmer, D. E. \& Kern, D. Beryllofluoride binding mimics phosphorylation of aspartate in response regulators. J. Bacteriol. 187, 8229-8230 (2005).

13. Liu, Y., Mao, X., Liu, M. \& Jiang, L. Impact of magnesium(II) on beryllium fluorides in solutions studied by ${ }^{19} \mathrm{~F}$ NMR spectroscopy. Chin. J. Chem. 32, 878-882 (2014).

14. Liu, Y., Mao, X., Liu, M. \& Jiang, L. Beryllium fluoride exchange rate accelerated by $\mathrm{Mg}^{2+}$ as discovered by ${ }^{19} \mathrm{~F}$ NMR. J. Phys. Chem. A. 119, 24-28 (2015).

15. Baxter, N. J. et al. Atomic details of near-transition state conformers for enzyme phosphoryl transfer revealed by $\mathrm{MgF}_{3}{ }^{-}$rather than by phosphoranes. Proc. Natl Acad. Sci. USA 107, 4555-4560 (2010).

16. Xiaoxia, L. et al. Prioritization of charge over geometry in transition state analogues of a dual specificity protein kinase. J. Am. Chem. Soc. 133, 3989-3994 (2011).

17. Kitevski-LeBlanc, J. L. \& Prosser, R. S. Current applications of ${ }^{19}$ F NMR to studies of protein structure and dynamics. Prog. Nucl. Mag. Res. Spectrosc. 62 1-33 (2012).

18. Zhu, Y., Qin, L., Yoshida, T. \& Inouye, M. Phosphatase activity of histidine kinase EnvZ without kinase catalytic domain. Proc. Natl Acad. Sci. USA 97, 7808-7813 (2000).

19. Dutta, R. \& Inouye, M. Reverse phosphotransfer from OmpR to EnvZ in a Kinase $^{-} /$Phosphatase ${ }^{+}$mutant of EnvZ (EnvZ $\left.\bullet N 347 D\right)$, a bifunctional signal transducer of Escherichia coli. J. Biol. Chem. 271, 1424-1429 (1996).

20. Lee, A. K., Detweiler, C. S. \& Falkow, S. OmpR regulates the two-component system SsrA-SsrB in Salmonella pathogenicity island 2. J. Bacteriol. 182, 771-781 (2000).

21. Garmendia, J., Beuzón, C. R., Ruiz-Albert, J. \& Holden, D. W. The roles of SsrA-SsrB and OmpR-EnvZ in the regulation of genes encoding the Salmonella typhimurium SPI-2 type III secretion system. Microbiology 149, 2385-2396 (2003).

22. Willett, J. W., Herrou, J., Briegel, A., Rotskoff, G. \& Crosson, S. Structural asymmetry in a conserved signaling system that regulates division, replication, and virulence of an intracellular pathogen. Proc. Natl Acad. Sci. USA 112, E3709-E3718 (2015).

23. Dubey, B. N. et al. Cyclic di-GMP mediates a histidine kinase/phosphatase switch by noncovalent domain cross-linking. Sci. Adv. 2, e1600823 (2016).

24. Sehgal, A. A., Duma, L., Bodenhausen, G. \& Pelupessy, P. Fast proton exchange in histidine: measurement of rate constants through indirect detection by NMR spectroscopy. Chem. Eur. J. 20, 6332-6338 (2014).

25. Podgornaia Anna, I., Casino, P., Marina, A. \& Laub Michael, T. Structural basis of a rationally rewired protein-protein interface critical to bacterial signaling. Structure 21, 1636-1647 (2013).

26. Casino, P., Fernandez-Alvarez, A., Alfonso, C., Rivas, G. \& Marina, A. Identification of a novel two component system in Thermotoga maritima. Complex stoichiometry and crystallization. Biochim. Biophys. Acta 1774, 603-609 (2007).

27. Anderson, K. M. et al. Direct observation of the ion-pair dynamics at a proteinDNA interface by NMR spectroscopy. J. Am. Chem. Soc. 135, 3613-3619 (2013).

28. Iwahara, J., Jung, Y.-S. \& Clore, G. M. Heteronuclear NMR spectroscopy for lysine $\mathrm{NH}_{3}$ groups in proteins: unique effect of water exchange on ${ }^{15} \mathrm{~N}$ transverse relaxation. J. Am. Chem. Soc. 129, 2971-2980 (2007).

29. Takayama, Y., Sahu, D. \& Iwahara, J. Observing in-phase single-quantum ${ }^{15} \mathrm{~N}$ multiplets for groups with two-dimensional heteronuclear correlation spectroscopy. J. Magn. Reson. 194, 313-316 (2008).

30. Delaglio, F. et al. NMRPipe: a multidimensional spectral processing system based on UNIX pipes. J. Biomol. NMR 6, 277-293 (1995).

31. Kabsch, W. XDS. Acta Crystallogr. D. 66, 125-132 (2010). 
32. Otwinowski, Z. \& Minor, W. Processing of X-ray diffraction data collected in oscillation mode. Methods Enzymol. 276, 307-326 (1997).

33. Strong, M. et al. Toward the structural genomics of complexes: Crystal structure of a PE/PPE protein complex from Mycobacterium tuberculosis. Proc. Natl Acad. Sci. USA 103, 8060-8065 (2006).

34. Collaborative Computational Project N. The CCP4 suite: programs for protein crystallography. Acta Crystallogr. D. 50, 760-763 (1994).

35. Adams, P. D. et al. PHENIX: building new software for automated crystallographic structure determination. Acta Crystallogr. D. 58, 1948-1954 (2002).

36. Emsley, P. \& Cowtan, K. Coot: model-building tools for molecular graphics. Acta Crystallogr. D. 60, 2126-2132 (2004).

37. Gao, R. \& Stock, A. M. Probing kinase and phosphatase activities of twocomponent systems in vivo with concentration-dependent phosphorylation profiling. Proc. Natl Acad. Sci. USA 110, 672-677 (2013).

38. McClelland, M. et al. Complete genome sequence of Salmonella enterica serovar Typhimurium LT2. Nature 413, 852-856 (2001).

39. Vikram, A. et al. Citrus flavonoid represses Salmonella pathogenicity island 1 and motility in S. Typhimurium LT2. Int. J. Food Microbiol. 145, 28-36 (2011).

40. Datsenko, K. A. \& Wanner, B. L. One-step inactivation of chromosomal genes in Escherichia coli K-12 using PCR products. Proc. Natl Acad. Sci. USA 97, 6640-6645 (2000).

41. $\mathrm{Hu}$, Y. et al. OmpR positively regulates urease expression to enhance acid survival of Yersinia pseudotuberculosis. Microbiology 155, 2522-2531 (2009).

\section{Acknowledgements}

We would like to thank Prof. Honggao Yan from the Michigan State University for providing the HK853 ${ }^{\mathrm{DHp}}$ and RR468 plasmids, Mr. Zhongwei Wang from the Wuhan Institute of Virology for assistance with the qRT-PCR experiment, Prof. Lin Guo and Dr. Lizhi Hu from the Wuhan University for help with the infectivity experiment, and Dr. Chenyu Cao from the Northwest A\&F University for optimizing the protocols of HK853 ${ }^{\text {DHp }}$ and RR468 purification. Use of the Advanced Photon Source was supported by the U.S. Department of Energy, Office of Science, Office of Basic Energy Sciences, under Contract No. W-31-109-Eng-38. This work was supported by grants from National Key R\&D Program of China (\#2017YFA0505400 awarded to Dr. Xu Zhang from Wuhan Institute of Physics and Mathematics), Ministry of Science and Technology of China (\#2013CB910200 awarded to Dr. Chun Tang from Wuhan Institute of Physics and Mathematics) and the Natural Science Foundation of China ( $\# 21573280$ awarded to L.J. and \#21603268 awarded to Y.L.) and the National Institute of General Medical Sciences (GM115355 awarded to P.Z.).

\section{Author contributions}

L.J., P.Z., and Y.L. designed the project. Y.L. performed the NMR sample preparation, NMR data collection, crystallization experiments of the wild-type $\mathrm{HK}_{85} 3^{\mathrm{CP}}-\mathrm{BeF}_{3}{ }^{-}-\mathrm{RR} 468$ complex and in vitro assays. J.R., Y.L., and P.Z. determined the crystal structure of the wild-type complex. J.R. performed the T264A $\mathrm{HK} 853^{\mathrm{cP}}$ mutation, sample preparation, crystallization. J.R. and P.Z. determined the structure of T264A HK853 $3^{\mathrm{C}} \mathrm{B}_{-} \mathrm{BeF}_{3}{ }^{-}$-RR468 complex. S.H. and Y.H. performed chromosome mutation and qRT-PCR experiments. Q.W. and Y.L. performed invasion experiments. D.W. helped with sample preparations. Y.L., L.J., P.Z., C.L., and M.L. analyzed the data. Y.L., L.J., and P.Z. wrote the manuscript with critical input from all authors.

\section{Additional information}

Supplementary Information accompanies this paper at https://doi.org/10.1038/s41467017-02310-9.

Competing interests: The authors declare no competing financial interests.

Reprints and permission information is available online at http://npg.nature.com/ reprintsandpermissions/

Publisher's note: Springer Nature remains neutral with regard to jurisdictional claims in published maps and institutional affiliations.

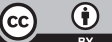

Open Access This article is licensed under a Creative Commons Attribution 4.0 International License, which permits use, sharing, adaptation, distribution and reproduction in any medium or format, as long as you give appropriate credit to the original author(s) and the source, provide a link to the Creative Commons license, and indicate if changes were made. The images or other third party material in this article are included in the article's Creative Commons license, unless indicated otherwise in a credit line to the material. If material is not included in the article's Creative Commons license and your intended use is not permitted by statutory regulation or exceeds the permitted use, you will need to obtain permission directly from the copyright holder. To view a copy of this license, visit http://creativecommons.org/ licenses/by/4.0/.

(C) The Author(s) 2017 